\title{
STANDARD DEVIATION OF THE GEODETIC QUADRILATERAL POINT COORDINATES DETERMINED BY THE APPLICATION OF HANSEN'S METHOD
}

\section{STANDARDNA ODSTUPANJA KOORDINATA TAČAKA GEODETSKOG ČETVOROUGLA ODREĐENIH PRIMENOM HANZENOVOG POSTUPKA}

\author{
Ganić Aleksandar ${ }^{1}$, Milutinović Aleksandar ${ }^{1}$, Gojković Zoran ${ }^{1}$, \\ Mikanović Radenko ${ }^{2}$, Vidanović Nebojša ${ }^{1}$
}

Received: May 25, 2015

Accepted: June 12, 2015

\begin{abstract}
The Danish astronomer Peter Andreas Hansen, who also worked on theoretical geodesy, provided an original solution for solving the geodetic quadrilateral in which the four angles and the length of one side are known. This procedure, identified in scientific literature as Hansen's problem, has been applied in geodesy but significantly more so when solving various problems related to mining measurements in underground exploitation. Nonetheless, the literature does not cover the determining of the standard deviation of the coordinates of unknown points which are established using Hansen's method. In this paper then, equations are derived to calculate the standard deviation of the coordinates determined by the above mentioned procedure.
\end{abstract}

Key words: geodetic quadrilateral, Hansen's problem, standard deviation

\begin{abstract}
Apstrakt: Danski astronom Peter Andreas Hansen, koji se bavio i teorijskom geodezijom dao je originalno rešenje za rešavanje geodetskog četvorougla $u$ kome su poznata četiri ugla i dužina jedne strane. Ovaj postupak koji se u stručnoj literaturi naziva "Hanzenov problem" našao je primenu u Geodeziji, ali znatno više u rešavanju različitih zadataka iz oblasti Rudarskih merenja koji se javljaju pri podzemnoj eksploataciji. Međutim, ono što u literaturi nije obrađeno predstavlja određivanje standardnih odstupanja koordinata nepoznatih tačaka do kojih se dolazi primenom Hanzenovog postupka. U ovom radu izvedene su jednačine za računanje standardnih odstupanja koordinata određenih navedenim postupkom.
\end{abstract}

Ključne reči: geodetski četvorougao, Hanzenov problem, standardno odstupanje

\footnotetext{
${ }^{1}$ University of Belgrade - Faculty of Minig and Geology, Đušina 7, 11000 Belgrade, Serbia,

e-mails: aleksandar.ganic@rgf.bg.ac.rs; aleksandar.milutinovic@rgf.bg.ac.rs; zoran.gojkovic@rgf.bg.ac.rs; nebojsa.vidanovic@rgf.bg.ac.rs

${ }^{2}$ University of Banja Luka - Faculty of Mine Engineering, Save Kovačevića bb, 79101 Prijedor, Republic of Srpska, Bosnia and Herzegovina; e-mail: radenko.mikanovic@unibl.rs
} 


\section{INTRODUCTION}

Peter Andreas Hansen (1795- 1874) was a Danish astronomer whose most famous work is in the field of celestial mechanics: theories on the motion of comets, small planets, the Moon, as well as lunar tables (Ephemerides). In addition to astronomy, Hansen worked on optics, probability theory and theoretical geodesy. In the field of Geodesy, he is well-known for his method of solving the geodetic quadrilateral in which four angles and the length of one side are known. Based on these elements, the unknown angles $\varphi$ and $\psi$ (Figure 1) are calculated, followed by all the other elements of the quadrilateral.

Such a way of solving the geodetic quadrilateral, known as Hansen's problem can be applied in:

- $\quad$ Determining the elements of eccentricity (Mihailović, 1987);

- Determining the base for terrestrial photogrammetric surveying (Schofield and Breach, 2007);

- $\quad$ Determining the points for vertical shaft surveys (Patarić, 1990);

- Connecting pits to the base on the surface of the terrain (Baturić, 1959), etc.

On the whole, Hansen's problem is applied in the instances where two points of the quadrilateral are inaccessible or are, for reasons of safety, inconvenient for stations at which it is necessary to focus the surveying instrument. The four angles required in the quadrilateral are measured only from the remaining two points of the quadrilateral.

When it is necessary to evaluate the coordinates of the points in the geodetic quadrilateral, then it is, as in every free trigonometrical network, essential to know the coordinates of the two points. In that case, the length of the side between these two points is calculated on the basis of the coordinates of the given points, while only angles are measured on the terrain. It follows that two situations are then feasible:

- $\quad$ The angles are measured from the given points, and

- The angles are measured from unknown points.

The first situation, where the angles are measured from the given points is more straightforward and does not involve calculating the elements of the geodetic quadrilateral. Based on the directional angle of the side between the given points and the measured angles, oriented directions are calculated in terms of unknown points or their coordinates (Chandra, 2005).

The second situation, when the angles are measured from unknown points, is more complex and requires that the geodetic quadrilateral be previously solved in accordance with Hansen's method.

The five known elements (four angles and one side) represent the mathematical minimum hence providing a unique solution. All additional measurements of an element of the geodetic quadrilateral (redundant measurements) offer an ambiguous solution necessitating the leveling of all measured values. In this case the calculations for the coordinates of unknown angles will be carried out by applying the methods of indirect leveling, thus obtaining the standard deviation of the coordinates of the unknown points. 


\section{CALCULATING ELEMENTS OF THE GEODETIC QUADRILATERAL USING HANSEN'S METHOD}

The geodetic quadrilateral problem which consists of two given but inaccessible points and two unknown but accessible points can be solved using Hansen's method (www2.washjeff).

Points $A$ and $B$ are given points with known coordinates $Y_{A}$ and $X_{A}$, or $Y_{B}$ and $X_{B}$, but they are inaccessible and angles cannot be measured from them. The horizontal length $d$ between them is also known and is calculated on the basis of the coordinates of these points.

In order to solve the quadrilateral or to determine the coordinates of the unknown but accessible points 1 and 2 it is sufficient to measure, on the terrain, the horizontal angles from these points: $\alpha_{1}, \alpha_{2}, \beta_{1}, \beta_{2}$.

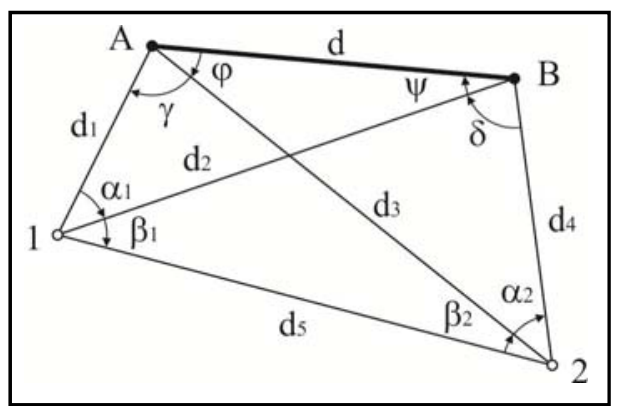

Figure 1 - Elements of the geodetic quadrilateral which is solved using Hansen's method

On account of measured and known (given) quantities, all unknown quantities in the quadrilateral are calculated: angles $\gamma, \delta, \varphi, \psi$ and lengths $d_{1}, d_{2}, d_{3}, d_{4}$ and $d_{5}$.

The unknown angles $\gamma$ and $\delta$ are derived from triangles:

from $\triangle \mathrm{A} 12: \quad \gamma=180^{\circ}-\alpha_{1}-\beta_{1}-\beta_{2}$

from $\Delta \mathrm{B} 12: \quad \delta=180^{\circ}-\alpha_{2}-\beta_{1}-\beta_{2}$

The unknown angles $\varphi$ and $\psi$ are calculated on the basis of their semi-sums and semi-differences, or:

$$
\begin{aligned}
& \varphi=\frac{\varphi+\psi}{2}+\frac{\varphi-\psi}{2} \\
& \psi=\frac{\varphi+\psi}{2}-\frac{\varphi-\psi}{2}
\end{aligned}
$$

Given that $\varphi+\psi=\beta_{1}+\beta_{2}$ (from $\Delta \mathrm{AB} 1$ and $\Delta \mathrm{A} 12$ ), the semi-sum of the unknown angles is:

$$
\frac{\varphi+\psi}{2}=\frac{\beta_{1}+\beta_{2}}{2}
$$

In accordance with the Sine rule: 


$$
\frac{d}{d_{1}}=\frac{\sin \alpha_{1}}{\sin \psi} ; \quad \frac{d_{1}}{d_{5}}=\frac{\sin \beta_{2}}{\sin \gamma} ; \quad \frac{d_{5}}{d_{4}}=\frac{\sin \delta}{\sin \beta_{1}} ; \quad \frac{d_{4}}{d}=\frac{\sin \varphi}{\sin \alpha_{2}}
$$

After multiplying:

$$
\frac{d}{d_{1}} \cdot \frac{d_{1}}{d_{5}} \cdot \frac{d_{5}}{d_{4}} \cdot \frac{d_{4}}{d}=1=\frac{\sin \alpha_{1} \cdot \sin \beta_{2} \cdot \sin \delta \cdot \sin \varphi}{\sin \psi \cdot \sin \gamma \cdot \sin \beta_{1} \cdot \sin \alpha_{2}}
$$

Or:

$$
\tan \mu=\frac{\sin \varphi}{\sin \psi}=\frac{\sin \gamma \cdot \sin \beta_{1} \cdot \sin \alpha_{2}}{\sin \alpha_{1} \cdot \sin \beta_{2} \cdot \sin \delta}
$$

As:

$$
\frac{\tan \frac{\varphi-\psi}{2}}{\tan \frac{\varphi+\psi}{2}}=\frac{\tan \mu-1}{1+\tan \mu}=\tan \left(\mu-45^{\circ}\right)
$$

The semi-difference of the unknown angles is:

$$
\frac{\varphi-\psi}{2}=\arctan \left[\tan \frac{\varphi+\psi}{2} \cdot \tan \left(\mu-45^{\circ}\right)\right]
$$

The unknown sides of the quadrilateral $d_{1}$ and $d_{4}$, as well as its diagonal $d_{2}$ and $d_{3}$, are calculated using the Sine rule based on the known side $d$ :

$$
\begin{aligned}
& d_{1}=\frac{\sin \psi}{\sin \alpha_{1}} \cdot d ; \quad d_{2}=\frac{\sin (\gamma+\varphi)}{\sin \alpha_{1}} \cdot d ; \\
& d_{3}=\frac{\sin (\delta+\psi)}{\sin \alpha_{2}} \cdot d ; \quad d_{4}=\frac{\sin \varphi}{\sin \alpha_{2}} \cdot d
\end{aligned}
$$

While side $d_{5}$ is calculated in terms of the previously evaluated sides (11):

$$
d_{5}=\frac{\sin \gamma}{\sin \beta_{2}} \cdot d_{1}=\frac{\sin \delta}{\sin \left(\alpha_{2}+\beta_{2}\right)} \cdot d_{2}=\frac{\sin \gamma}{\sin \left(\alpha_{1}+\beta_{1}\right)} \cdot d_{3}=\frac{\sin \delta}{\sin \beta_{1}} \cdot d_{4}
$$

The coordinates of the unknown points 1 and 2 are calculated on the basis of the evaluated elements of the quadrilateral, in terms of the known points $A$ and $B$, using the equations:

$$
\begin{aligned}
& Y_{1}=Y_{A}+d_{1} \cdot \sin v_{A}^{1}=Y_{A}+d_{1} \cdot \sin \left(v_{A}^{B}+\varphi+\gamma\right) \\
& X_{1}=X_{A}+d_{1} \cdot \cos v_{A}^{1}=X_{A}+d_{1} \cdot \cos \left(v_{A}^{B}+\varphi+\gamma\right)
\end{aligned}
$$

Or

$$
\begin{aligned}
& Y_{1}=Y_{B}+d_{2} \cdot \sin v_{B}^{1}=Y_{B}+d_{2} \cdot \sin \left(v_{B}^{A}-\psi\right) \\
& X_{1}=X_{B}+d_{2} \cdot \cos v_{B}^{1}=X_{B}+d_{2} \cdot \cos \left(v_{B}^{A}-\psi\right)
\end{aligned}
$$

As:

$$
\begin{aligned}
& Y_{2}=Y_{B}+d_{4} \cdot \sin v_{B}^{2}=Y_{B}+d_{4} \cdot \sin \left(v_{B}^{A}-\psi-\delta\right) \\
& X_{2}=X_{B}+d_{4} \cdot \cos v_{B}^{2}=X_{B}+d_{4} \cdot \cos \left(v_{B}^{A}-\psi-\delta\right)
\end{aligned}
$$

Or 


$$
\begin{aligned}
& Y_{2}=Y_{A}+d_{3} \cdot \sin v_{A}^{2}=Y_{A}+d_{3} \cdot \sin \left(v_{A}^{B}+\varphi\right) \\
& X_{2}=X_{A}+d_{3} \cdot \cos v_{A}^{2}=X_{A}+d_{3} \cdot \cos \left(v_{A}^{B}+\varphi\right)
\end{aligned}
$$

\section{STANDARD DEVIATION OF THE COORDINATES OF UNKNOWN POINTS}

The standard deviation of the coordinates of unknown points 1 and 2, due to extensive deriving, will be evaluated only on the basis of equations (13) and (15).

Previously, in these equations, all evaluated values will be expressed only in terms of measured angles $\alpha_{1}, \alpha_{2}, \beta_{1}$ and $\beta_{2}$ :

$$
\begin{aligned}
& Y_{1}=Y_{A}+\sin \left[v_{A}^{B}+(B+A)+\left(180^{\circ}-\alpha_{1}-\beta_{1}-\beta_{2}\right)\right] \cdot \frac{\sin (B-A)}{\sin \alpha_{1}} \cdot d \\
& X_{1}=X_{A}+\cos \left[v_{A}^{B}+(B+A)+\left(180^{\circ}-\alpha_{1}-\beta_{1}-\beta_{2}\right)\right] \cdot \frac{\sin (B-A)}{\sin \alpha_{1}} \cdot d \\
& Y_{2}=Y_{B}+\sin \left[v_{B}^{A}-(B-A)-\left(180^{\circ}-\alpha_{2}-\beta_{1}-\beta_{2}\right)\right] \cdot \frac{\sin (B+A)}{\sin \alpha_{2}} \cdot d \\
& X_{2}=X_{B}+\cos \left[v_{B}^{A}-(B-A)-\left(180^{\circ}-\alpha_{2}-\beta_{1}-\beta_{2}\right)\right] \cdot \frac{\sin (B+A)}{\sin \alpha_{2}} \cdot d
\end{aligned}
$$

Where shifts are introduced:

$A=\arctan \left(\frac{\sin \left(\alpha_{1}+\beta_{1}+\beta_{2}\right) \cdot \sin \alpha_{2} \cdot \sin \beta_{1}-\sin \left(\alpha_{2}+\beta_{1}+\beta_{2}\right) \cdot \sin \alpha_{1} \cdot \sin \beta_{2}}{\sin \left(\alpha_{1}+\beta_{1}+\beta_{2}\right) \cdot \sin \alpha_{2} \cdot \sin \beta_{1}+\sin \left(\alpha_{2}+\beta_{1}+\beta_{2}\right) \cdot \sin \alpha_{1} \cdot \sin \beta_{2}} \frac{\beta_{1}+\beta_{2}}{2}\right)$

$B=\frac{\beta_{1}+\beta_{2}}{2}$

The standard deviation of the coordinates of unknown points 1 and 2 will be calculated only on the basis of standard deviations of measured angles $\alpha_{1}, \alpha_{2}, \beta_{1}$ and $\beta_{2}$, while the coordinates of the given points $A$ and $B$, as well as the value of the grid bearing $v_{A}^{B}$ and the length $d$ will be considered true values.

In accordance with that, the standard deviation of point coordinates will be calculated using the equations:

$$
\begin{aligned}
& \sigma_{Y_{1}}=\sqrt{\left(\frac{\partial Y_{1}}{\partial \alpha_{1}}\right)^{2} \cdot\left(\frac{\sigma_{\alpha_{1}}}{\rho^{\prime \prime}}\right)^{2}+\left(\frac{\partial Y_{1}}{\partial \alpha_{2}}\right)^{2} \cdot\left(\frac{\sigma_{\alpha_{2}}}{\rho^{\prime \prime}}\right)^{2}+\left(\frac{\partial Y_{1}}{\partial \beta_{1}}\right)^{2} \cdot\left(\frac{\sigma_{\beta_{1}}}{\rho^{\prime \prime}}\right)^{2}+\left(\frac{\partial Y_{1}}{\partial \beta_{2}}\right)^{2} \cdot\left(\frac{\sigma_{\beta_{2}}}{\rho^{\prime \prime}}\right)^{2}} \\
& \sigma_{X_{1}}=\sqrt{\left(\frac{\partial X_{1}}{\partial \alpha_{1}}\right)^{2} \cdot\left(\frac{\sigma_{\alpha_{1}}}{\rho^{\prime \prime}}\right)^{2}+\left(\frac{\partial X_{1}}{\partial \alpha_{2}}\right)^{2} \cdot\left(\frac{\sigma_{\alpha_{2}}}{\rho^{\prime \prime}}\right)^{2}+\left(\frac{\partial X_{1}}{\partial \beta_{1}}\right)^{2} \cdot\left(\frac{\sigma_{\beta_{1}}}{\rho^{\prime \prime}}\right)^{2}+\left(\frac{\partial X_{1}}{\partial \beta_{2}}\right)^{2} \cdot\left(\frac{\sigma_{\beta_{2}}}{\rho^{\prime \prime}}\right)^{2}} \\
& \sigma_{Y_{2}}=\sqrt{\left(\frac{\partial Y_{2}}{\partial \alpha_{1}}\right)^{2} \cdot\left(\frac{\sigma_{\alpha_{1}}}{\rho^{\prime \prime}}\right)^{2}+\left(\frac{\partial Y_{2}}{\partial \alpha_{2}}\right)^{2} \cdot\left(\frac{\sigma_{\alpha_{2}}}{\rho^{\prime \prime}}\right)^{2}+\left(\frac{\partial Y_{2}}{\partial \beta_{1}}\right)^{2} \cdot\left(\frac{\sigma_{\beta_{1}}}{\rho^{\prime \prime}}\right)^{2}+\left(\frac{\partial Y_{2}}{\partial \beta_{2}}\right)^{2} \cdot\left(\frac{\sigma_{\beta_{2}}}{\rho^{\prime \prime}}\right)^{2}}
\end{aligned}
$$


$\sigma_{X_{2}}=\sqrt{\left(\frac{\partial X_{2}}{\partial \alpha_{1}}\right)^{2} \cdot\left(\frac{\sigma_{\alpha_{1}}}{\rho^{\prime \prime}}\right)^{2}+\left(\frac{\partial X_{2}}{\partial \alpha_{2}}\right)^{2} \cdot\left(\frac{\sigma_{\alpha_{2}}}{\rho^{\prime \prime}}\right)^{2}+\left(\frac{\partial X_{2}}{\partial \beta_{1}}\right)^{2} \cdot\left(\frac{\sigma_{\beta_{1}}}{\rho^{\prime \prime}}\right)^{2}+\left(\frac{\partial X_{2}}{\partial \beta_{2}}\right)^{2} \cdot\left(\frac{\sigma_{\beta_{2}}}{\rho^{\prime \prime}}\right)^{2}}$

Where:

$\sigma_{\alpha_{1}} ; \sigma_{\alpha_{2}} ; \sigma_{\beta_{1}} ; \sigma_{\beta_{2}}$ - standard deviations of measured angles;

and partial derivatives of functions are:

$$
\begin{aligned}
& \frac{\partial Y_{1}}{\partial \alpha_{1}}=d \cdot \csc \alpha_{1}\left\{-\cot \alpha_{1} \cdot \sin \left(v_{A}^{B}+180^{\circ}-\alpha_{1}+A-B\right) \cdot \sin (B-A)-\right. \\
& -\frac{\sin \left(\alpha_{2}+\beta_{2}\right) \cdot \sin ^{2} B \cdot \sin (B-A) \cdot \cos \left(v_{A}^{B}+180^{\circ}-\alpha_{1}+A-B\right)}{C\left(\sec ^{2} B\right)(C+2 D \cos 2 B)+D^{2}\left(1+\tan ^{2} B\right)} . \\
& \frac{\left[\sin \left(2 \alpha_{1}-\alpha_{2}-\beta_{2}\right)-\sin \left(2 \alpha_{1}-\alpha_{2}+\beta_{2}\right)+2 \sin \left(\alpha_{2}+\beta_{2}\right)+\sin \left(2 \alpha_{1}-\alpha_{2}+2 \beta_{1}+\beta_{2}\right)-\sin \left(2 \alpha_{1}+\alpha_{2}+2 \beta_{1}+\beta_{2}\right)\right]}{C\left(\sec ^{2} B\right)(C+2 D \cos 2 B)+D^{2}\left(1+\tan ^{2} B\right)}+ \\
& \left.+\frac{\sin \left(v_{A}^{B}+180^{\circ}-\alpha_{1}+A-B\right) \cdot \sin \alpha_{2} \cdot \sin \beta_{1} \cdot \sin \beta_{2} \cdot \sin \left(\alpha_{2}+2 B\right) \cdot \sin 2 B \cdot 2 \cos (B-A) \cdot \tan B}{C\left(\sec ^{2} B\right)(C+2 D \cos 2 B)+D^{2}\left(1+\tan ^{2} B\right)}\right\}
\end{aligned}
$$

$\frac{\partial Y_{1}}{\partial \alpha_{2}}=2 d\left[\frac{\sin \beta_{1} \cdot \sin \beta_{2} \cdot \sin 2 B \cdot \sin \left(\alpha_{1}+2 B_{1}\right) \cdot \tan B}{C\left(\sec ^{2} B\right)(C+2 D \cos 2 B)+D^{2}\left(1+\tan ^{2} B\right)}\right.$.

$\left.\frac{\sin (B-A) \cdot \cos \left(v_{A}^{B}+180^{\circ}-\alpha_{1}+A-B\right)-\cos (B-A) \cdot \sin \left(\nu_{A}^{B}+180^{\circ}-\alpha_{1}+A-B\right)}{C\left(\sec ^{2} B\right)(C+2 D \cos 2 B)+D^{2}\left(1+\tan ^{2} B\right)}\right]$

$\frac{\partial Y_{1}}{\partial \beta_{1}}=-4 d\left[\frac{\sin \left(\alpha_{1}-\alpha_{2}\right) \cdot \sin \beta_{2} \cdot \sin \left(\alpha_{2}+\beta_{2}\right) \cdot \sin ^{2} B}{C \cdot\left(\sec ^{2} B\right)(C+2 D \cos 2 B)+D^{2}\left(1+\tan ^{2} B\right)}\right.$.

$\left.\frac{\sin (B-A) \cdot \cos \left(v_{A}^{B}+180^{\circ}-\alpha_{1}+A-B\right)-\cos (B-A) \cdot \sin \left(v_{A}^{B}+180^{\circ}-\alpha_{1}+A-B\right)}{C \cdot\left(\sec ^{2} B\right)(C+2 D \cos 2 B)+D^{2}\left(1+\tan ^{2} B\right)}\right]$

$\frac{\partial Y_{1}}{\partial \beta_{2}}=d\left\{\frac{\sin ^{2} B\left[-2 \sin \alpha_{1}+\sin \left(\alpha_{1}+2 \beta_{2}\right)+\sin \left(\alpha_{1}-2 \alpha_{2}-2 \beta_{2}\right)-\sin \left(\alpha_{1}+4 B\right)+\sin \left(\alpha_{1}+2 \alpha_{2}+4 B\right)\right]}{C\left(\sec ^{2} B\right)(C+2 D \cos 2 B)+D^{2}\left(1+\tan ^{2} B\right)}\right.$.

$\left.\frac{\sin (B-A) \cdot \cos \left(v_{A}^{B}+180^{\circ}-\alpha_{1}+A-B\right)-\cos (B-A) \cdot \sin \left(v_{A}^{B}+180^{\circ}-\alpha_{1}+A-B\right)}{C\left(\sec ^{2} B\right)(C+2 D \cos 2 B)+D^{2}\left(1+\tan ^{2} B\right)}\right\}$

$\frac{\partial X_{1}}{\partial \alpha_{1}}=d \cdot \csc \alpha_{1}\left\{-\cos \left(v_{A}^{B}+180^{\circ}-\alpha_{1}+A-B\right) \cdot \sin (B-A) \cdot \cot \alpha_{1}+\frac{\sin \left(\alpha_{2}+\beta_{2}\right) \cdot \sin ^{2} B \cdot \sin (B-A)}{C\left(\sec ^{2} B\right)(C+2 D \cos 2 B)+D^{2}\left(1+\tan ^{2} B\right)} \cdot\right.$

$+\frac{\left[\sin \left(2 \alpha_{1}-\alpha_{2}-\beta_{2}\right)-\sin \left(2 \alpha_{1}-\alpha_{2}+\beta_{2}\right)+2 \sin \left(\alpha_{2}+\beta_{2}\right)+\sin \left(2 \alpha_{1}-\alpha_{2}+2 \beta_{1}+\beta_{2}\right)-\sin \left(2 \alpha_{1}+\alpha_{2}+2 \beta_{1}+\beta_{2}\right)\right]}{C\left(\sec ^{2} B\right)(C+2 D \cos 2 B)+D^{2}\left(1+\tan ^{2} B\right)}+$

$\left.+\frac{2 \cos (B-A) \cdot \cos \left(v_{A}^{B}+180^{\circ}-\alpha_{1}+A-B\right) \cdot \sin \alpha_{2} \cdot \sin \beta_{1} \cdot \sin \beta_{2} \cdot \sin \left(\alpha_{2}+2 B\right) \cdot \sin 2 B \cdot \tan B}{C\left(\sec ^{2} B\right)(C+2 D \cos 2 B)+D^{2}\left(1+\tan ^{2} B\right)}\right\}$ 


$$
\begin{aligned}
& \frac{\partial X_{1}}{\partial \alpha_{2}}=-2 d\left[\frac{\sin \left(\alpha_{1}+2 B\right) \cdot \sin \beta_{1} \cdot \sin \beta_{2} \cdot \sin 2 B \cdot \tan B}{C\left(\sec ^{2} B\right)(C+2 D \cos 2 B)+D^{2}\left(1+\tan ^{2} B\right)} \cdot\right. \\
& \left.\frac{\cos (B-A) \cdot \cos \left(v_{A}^{B}+180^{\circ}-\alpha_{1}+A-B\right)-\sin (B-A) \cdot \sin \left(v_{A}^{B}+180^{\circ}-\alpha_{1}+A-B\right)}{C\left(\sec ^{2} B\right)(C+2 D \cos 2 B)+D^{2}\left(1+\tan ^{2} B\right)}\right] \\
& \frac{\partial X_{1}}{\partial \beta_{1}}=4 d\left[\frac{\sin \left(\alpha_{1}-\alpha_{2}\right) \cdot \sin \left(\alpha_{2}+\beta_{2}\right) \cdot \sin \beta_{2} \cdot \sin ^{2} B}{C\left(\sec ^{2} B\right)(C+2 D \cos 2 B)+D^{2}\left(1+\tan ^{2} B\right)} \cdot\right. \\
& \left.\cdot \frac{\cos (B-A) \cdot \cos \left(v_{A}^{B}+180^{\circ}-\alpha_{1}+A-B\right)+\sin (B-A) \cdot \sin \left(v_{A}^{B}+180^{\circ}-\alpha_{1}+A-B\right)}{C\left(\sec ^{2} B\right)(C+2 D \cos 2 B)+D^{2}\left(1+\tan ^{2} B\right)}\right]
\end{aligned}
$$

$\frac{\partial X_{1}}{\partial \beta_{2}}=-d\left\{\frac{\sin ^{2} B\left[-2 \sin \alpha_{1}+\sin \left(\alpha_{1}+2 \beta_{2}\right)+\sin \left(\alpha_{1}-2 \alpha_{2}-2 \beta_{2}\right)-\sin \left(\alpha_{1}+4 B\right)+\sin \left(\alpha_{1}+2 \alpha_{2}+4 B\right)\right]}{C\left(\sec ^{2} B\right)(C+2 D \cos 2 B)+D^{2}\left(1+\tan ^{2} B\right)}\right.$.

$\left.\frac{\cos (B-A) \cdot \cos \left(v_{A}^{B}+180^{\circ}-\alpha_{1}+A-B\right)-\sin (B-A) \cdot \sin \left(v_{A}^{B}+180^{\circ}-\alpha_{1}+A-B\right)}{C\left(\sec ^{2} B\right)(C+2 D \cos 2 B)+D^{2}\left(1+\tan ^{2} B\right)}\right\}$

$\frac{\partial Y_{2}}{\partial \alpha_{1}}=2 d\left[\frac{\sin \beta_{1} \cdot \sin \beta_{2} \cdot \sin 2 B \cdot \sin \left(\alpha_{2}+2 B\right) \cdot \sin \left(180^{\circ}-v_{B}^{A}-\alpha_{2}-2 A-2 B\right) \cdot \tan B}{C\left(\sec ^{2} B\right)(C+2 D \cos 2 B)+D^{2}\left(1+\tan ^{2} B\right)}\right]$

$\frac{\partial Y_{2}}{\partial \alpha_{2}}=d \csc \alpha_{2}\left\{\sin \left(180^{\circ}-v_{B}^{A}-\alpha_{2}-A-B\right) \cdot \cot \alpha_{2} \cdot \sin (A+B)-\right.$

$-\frac{\left[-\sin \left(\alpha_{1}-2 \alpha_{2}-\beta_{1}\right)-2 \sin \left(\alpha_{1}+\beta_{1}\right)+\sin \left(\alpha_{1}-2 \alpha_{2}+\beta_{1}\right)+\sin \left(\alpha_{1}-2 \alpha_{2}-\beta_{1}-2 \beta_{2}\right)+\sin \left(\alpha_{1}+2 \alpha_{2}+\beta_{1}+2 \beta_{2}\right)\right]}{C\left(\sec ^{2} B\right)(C+2 D \cos 2 B)+D^{2}\left(1+\tan ^{2} B\right)}$.

$\frac{\sin \left(\alpha_{1}+\beta_{1}\right) \cdot \sin ^{2} B \cdot \cos \left(180^{\circ}-v_{B}^{A}-\alpha_{2}-A-B\right) \cdot \sin (A+B)}{C\left(\sec ^{2} B\right)(C+2 D \cos 2 B)+D^{2}\left(1+\tan ^{2} B\right)}-$

$\left.-\frac{\sin \left(180^{\circ}-v_{B}^{A}-\alpha_{2}-A-B\right) \cdot 2 \cos (A+B) \cdot \sin \alpha_{1} \cdot \sin \beta_{1} \cdot \sin \beta_{2} \cdot \sin \left(\alpha_{1}+2 B\right) \cdot \sin 2 B \cdot \tan B}{C\left(\sec ^{2} B\right)(C+2 D \cos 2 B)+D^{2}\left(1+\tan ^{2} B\right)}\right\}$

$$
\begin{aligned}
& \frac{\partial Y_{2}}{\partial \beta_{1}}=d\left\{\frac{\left[-2+2 \cos \left(2 \alpha_{2}\right)+\cos \left(2 \beta_{1}\right)+\cos \left(2 \alpha_{1}+2 \beta_{1}\right)-\cos \left(2 \alpha_{1}-2 \alpha_{2}+2 \beta_{1}\right)-\cos \left(2 \alpha_{2}+2 \beta_{1}\right)-\cos 4 B+\right.}{2\left[C\left(\sec ^{2} B\right)(C+2 D \cos 2 B)+D^{2}\left(1+\tan ^{2} B\right)\right]}\right. \\
& \left.\frac{\left.+\cos \left(2 \alpha_{1}+4 B\right)+\cos \left(2 \alpha_{2}+4 B\right)-\cos \left(2 \alpha_{1}+2 \alpha_{2}+4 B\right)\right] \cdot \csc ^{2} \alpha_{2} \cdot \sin ^{2} B \cdot \sin \left(180^{\circ}-v_{B}^{A}-\alpha_{2}-2 A-2 B\right)}{2\left[C\left(\sec ^{2} B\right)(C+2 D \cos 2 B)+D^{2}\left(1+\tan ^{2} B\right)\right]}\right\}
\end{aligned}
$$

$\frac{\partial Y_{2}}{\partial \beta_{2}}=4 d\left[\frac{\sin \left(\alpha_{1}-\alpha_{2}\right) \cdot \sin \left(\beta_{1}\right) \cdot \sin \left(\alpha_{1}+\beta_{1}\right) \cdot \sin ^{2} B \cdot \sin \left(180^{\circ}-v_{B}^{A}-\alpha_{2}-2 A-2 B\right)}{C\left(\sec ^{2} B\right)(C+2 D \cos 2 B)+D^{2}\left(1+\tan ^{2} B\right)}\right]$

$\frac{\partial X_{2}}{\partial \alpha_{1}}=-2 d\left[\frac{\sin \beta_{1} \cdot \sin \beta_{2} \cdot \sin 2 B \cdot \sin \left(\alpha_{2}+2 B\right) \cdot \tan B \cdot \cos \left(180^{\circ}-v_{B}^{A}-\alpha_{2}-2 A-2 B\right)}{C\left(\sec ^{2} B\right)(C+2 D \cos 2 B)+D^{2}\left(1+\tan ^{2} B\right)}\right]$ 
$\frac{\partial X_{2}}{\partial \alpha_{2}}=d \csc \alpha_{2}\left\{-\cos \left(180^{\circ}-v_{B}^{A}-\alpha_{2}-A-B\right) \cdot \cot \alpha_{2} \cdot \sin (A+B)-\right.$

$-\frac{\left[-\sin \left(\alpha_{1}-2 \alpha_{2}-\beta_{1}\right)-2 \sin \left(\alpha_{1}+\beta_{1}\right)+\sin \left(\alpha_{1}-2 \alpha_{2}+\beta_{1}\right)+\sin \left(\alpha_{1}-2 \alpha_{2}-\beta_{1}-2 \beta_{2}\right)+\sin \left(\alpha_{1}+2 \alpha_{2}+\beta_{1}+2 \beta_{2}\right)\right]}{C\left(\sec ^{2} B\right)(C+2 D \cos 2 B)+D^{2}\left(1+\tan ^{2} B\right)}$.

$\frac{\sin \left(\alpha_{1}+\beta_{1}\right) \cdot \sin ^{2} B \cdot \sin \left(180^{\circ}-v_{B}^{A}-\alpha_{2}-A-B\right) \cdot \sin (A+B)}{C\left(\sec ^{2} B\right)(C+2 D \cos 2 B)+D^{2}\left(1+\tan ^{2} B\right)}+$

$\left.+\frac{2 \cos \left(180^{\circ}-v_{B}^{A}-\alpha_{2}-A-B\right) \cdot \cos (A+B) \cdot \sin \alpha_{1} \cdot \sin \beta_{1} \cdot \sin \beta_{2} \cdot \sin 2 B \cdot \sin \left(\alpha_{1}+2 B\right) \cdot \tan B}{C\left(\sec ^{2} B\right)(C+2 D \cos 2 B)+D^{2}\left(1+\tan ^{2} B\right)}\right\}$

$\frac{\partial X_{2}}{\partial \beta_{1}}=d\left\{\frac{\left[2-2 \cos \left(2 \alpha_{2}\right)-\cos \left(2 \beta_{1}\right)-\cos \left(2 \alpha_{1}+2 \beta_{1}\right)+\cos \left(2 \alpha_{1}-2 \alpha_{2}+2 \beta_{1}\right)+\cos \left(2 \alpha_{2}+2 \beta_{1}\right)+\cos 4 B-\right.}{2\left[C\left(\sec ^{2} B\right)(C+2 D \cos 2 B)+D^{2}\left(1+\tan ^{2} B\right)\right]}\right.$

$\left.\frac{\left.-\cos \left(2 \alpha_{1}+4 B\right)-\cos \left(2 \alpha_{2}+4 B\right)+\cos \left(2 \alpha_{1}+2 \alpha_{2}+4 B\right)\right] \cdot \csc ^{2} \alpha_{2} \cdot \sin ^{2} B \cdot \cos \left(180^{\circ}-v_{B}^{A}-\alpha_{2}-2 A-2 B\right)}{2\left[C\left(\sec ^{2} B\right)(C+2 D \cos 2 B)+D^{2}\left(1+\tan ^{2} B\right)\right]}\right\}$

$\frac{\partial X_{2}}{\partial \beta_{2}}=-4 d\left[\frac{\sin \left(\alpha_{1}-\alpha_{2}\right) \cdot \sin \left(\beta_{1}\right) \cdot \sin \left(\alpha_{1}+\beta_{1}\right) \cdot \sin ^{2} B \cdot \cos \left(180^{\circ}-v_{B}^{A}-\alpha_{2}-2 A-2 B\right)}{C\left(\sec ^{2} B\right)(C+2 D \cos 2 B)+D^{2}\left(1+\tan ^{2} B\right)}\right]$

Where shifts are:

$C=\sin \alpha_{2} \cdot \sin \beta_{1} \cdot \sin \left(\alpha_{1}+\beta_{1}+\beta_{2}\right)$

$D=\sin \alpha_{1} \cdot \sin \beta_{2} \cdot \sin \left(\alpha_{2}+\beta_{1}+\beta_{2}\right)$

\subsection{Example}

An example of the analysis pertaining to the standard deviation of unknown points has been performed on the geodetic quadrilateral in the shape of a regular square whose elements are shown in Figure 2.

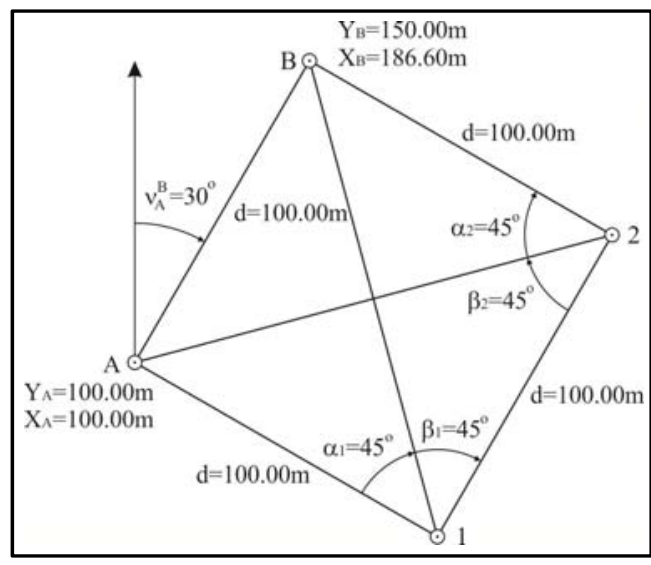

Figure 2 - Geodetic quadrilateral in the shape of a regular square 
The coordinates of unknown points 1 and 2 have been evaluated using equations (13) and (15):

$$
\begin{aligned}
& Y_{1}=186.60 \mathrm{~m} ; \quad X_{1}=50.00 \mathrm{~m} \\
& Y_{2}=236.60 \mathrm{~m} ; \quad X_{2}=136.60 \mathrm{~m}
\end{aligned}
$$

The values of the partial derivatives are:

$$
\begin{aligned}
& \frac{\partial Y_{1}}{\partial \alpha_{1}}=+100 \mathrm{~m} ; \quad \frac{\partial Y_{1}}{\partial \alpha_{2}}=-136.603 \mathrm{~m} ; \quad \frac{\partial Y_{1}}{\partial \beta_{1}}=0 ; \quad \frac{\partial Y_{1}}{\partial \beta_{2}}=+136.603 \mathrm{~m} \\
& \frac{\partial X_{1}}{\partial \alpha_{1}}=+173.206 \mathrm{~m} ; \quad \frac{\partial X_{1}}{\partial \alpha_{2}}=-36.603 \mathrm{~m} ; \quad \frac{\partial X_{1}}{\partial \beta_{1}}=0 ; \quad \frac{\partial X_{1}}{\partial \beta_{2}}=+36.603 \mathrm{~m} \\
& \frac{\partial Y_{2}}{\partial \alpha_{1}}=-36.603 \mathrm{~m} ; \quad \frac{\partial Y_{2}}{\partial \alpha_{2}}=-100 \mathrm{~m} ; \quad \frac{\partial Y_{2}}{\partial \beta_{1}}=+36,603 \mathrm{~m} ; \quad \frac{\partial Y_{2}}{\partial \beta_{2}}=0 \\
& \frac{\partial X_{2}}{\partial \alpha_{1}}=+136.603 \mathrm{~m} ; \frac{\partial X_{2}}{\partial \alpha_{2}}=-173.206 \mathrm{~m} ; \frac{\partial X_{2}}{\partial \beta_{1}}=-136.603 \mathrm{~m} ; \frac{\partial X_{2}}{\partial \beta_{2}}=0
\end{aligned}
$$

Based on these values and assuming various standard deviations of the measured angles using the equations (21-24), the standard deviations of the coordinates of the points 1 and 2 have been evaluated, where:

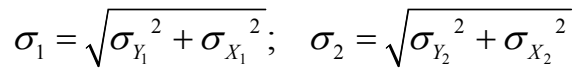

Table 1- Standard deviation of the coordinates of unknown points

\begin{tabular}{|cc||c|c|c|c|c|c|c|c|}
\hline \multicolumn{1}{|l||}{} & \multicolumn{8}{c|}{$\sigma_{\alpha_{1}}=\sigma_{\alpha_{2}}=\sigma_{\beta_{1}}=\sigma_{\beta_{2}}$} \\
& & $\mathbf{1 "}$ & $\mathbf{2 "}$ & $\mathbf{3 "}$ & $\mathbf{5}^{\prime \prime}$ & $\mathbf{1 0 "}$ & $\mathbf{2 0 "}$ & $\mathbf{3 0 "}$ & $\mathbf{6 0 "}$ \\
\hline \hline$\sigma_{Y_{1}}$ & {$[\mathbf{m m}]$} & 1.1 & 2.1 & 3.2 & 5.3 & 10.5 & 21.1 & 31.6 & 63.3 \\
\hline$\sigma_{X_{1}}$ & {$[\mathbf{m m}]$} & 0.9 & 1.8 & 2.6 & 4.4 & 8.8 & 17.5 & 26.3 & 52.6 \\
\hline$\sigma_{1}$ & {$[\mathbf{m m}]$} & 1.4 & 2.7 & 4.1 & 6.9 & 13.7 & 27.4 & 41.1 & 82.3 \\
\hline$\sigma_{Y_{2}}$ & {$[\mathbf{m m}]$} & 0.5 & 1.1 & 1.6 & 2.7 & 5.5 & 10.9 & 16.4 & 32.8 \\
\hline$\sigma_{X_{2}}$ & {$[\mathbf{m m}]$} & 1.3 & 2.5 & 3.8 & 6.3 & 12.6 & 25.2 & 37.7 & 75.5 \\
\hline$\sigma_{2}$ & {$[\mathbf{m m}]$} & 1.4 & 2.7 & 4.1 & 6.9 & 13.7 & 27.4 & 41.1 & 82.3 \\
\hline
\end{tabular}

As Table 1 shows, considering the regular shape of the geodetic quadrilateral, the standard deviations of the unknown points are mutually equal, and their value increases proportional to the decrease in the accuracy of the angle measurements in the quadrilateral.

\section{CONCLUSION}

The standard deviation of the coordinates of unknown points which is determined using Hansen's method is not presented in scientific literature. One reason for this, among others, must lie in the fact that it involves extensive and complex calculations, primarily of the coefficients which represent partial derivatives with respect to variable or measured quantities. Although contemporary measuring 
techniques enable the determining of the coordinates of any point providing there is mutual visibility, there is a whole range of different situations on the terrain which require the application of Hansen's method. This is, above all, the case when solving specific engineering problems in regard to mines with underground exploitation.

For this reason, it is vital to perceive the optimal shape of the geodetic quadrilateral, having in mind that errors in point coordinates, besides being influenced by errors in the measured angles, are also impacted by the shape of the quadrilateral. This paper serves as the first step in the analysis of the impact of the geodetic quadrilateral shape on the standard deviation of the coordinates of the unknown points where standard deviation equations have been derived.

\section{ACKNOWLEDGEMENT}

This paper was realized as a part of the project "Study of Possibilities for Valorization of the remaining Coal Reserves to Provide Stability of the Energy Sector of Republic of Serbia" (TR 33029) financed by the Ministry of Education, Science and Techonological Development of the Republic of Serbia within the framework of Programme of research in the field of technological development for the period 20112015.

\section{REFERENCES}

[1] BATURIĆ, J. (1959) Rudarska mjerenja II dio. Zagreb: Tehnička knjiga.

[2] CHANDRA, A.M. (2005) Surveying. New Delhi: New Age International (P) Ltd.

[3] MIHAILOVIĆ, K. (1987) Geodezija II, I deo. Beograd: Naučna knjiga.

[4] PATARIĆ, M. (1990) Rudarska merenja I deo. Beograd: Rudarsko-geološki fakultet.

[5] SCHOFIELD, W. and BREACH, M. (2007) Engineering Surveying. Oxford: Elsevier.

[6] Two Survey Problems [Online]. Available from: http://www2.washjeff.edu/users/mwoltermann/Dorrie/40.pdf [Accessed 12/03/15]. 